\title{
An Astronomer's View on the Current College-Level Textbook Descriptions of Tides
}

\author{
Kyungjin Ahn* \\ Department of Earth Science Education, Chosun University, Gwangju 501-759, Korea
}

\begin{abstract}
In the equilibrium theory of tides by Newton, tide on the Earth is a phenomenon driven by differential gravity contributed both by the Sun and the Moon. Due to the direct link of the generic tidal effect to the oceanic tides, college students in the earth science education department are exposed to this theory through oceanography lectures as well as astronomy lectures. Common oceanography textbooks adopt a non-inertial reference frame fixed to the Earth in which the fictitious, centrifugal force appears. This has a potential risk to provide misconceptions among students in various aspects including the followings: 1) this is how Newton originally derived the equilibrium theory of tides, and 2) the tide is a phenomenon appearing only in rotating systems. We show that in astronomy, a much simpler description, which employs the inertial frame, is generally used to explain tides and thus causes less confusion. We argue that the description used in astronomy is preferable both in the viewpoints of simplicity and ease of interpretation. Moreover, on a historical basis, an inertial frame was adopted by Newton in Principia to explain tides. Thus, the description used in astronomy is consistent with Newton's original approach. We also present various astrophysical tides which do not comply with the concept of centrifugal force in general. We therefore argue that the description used in oceanography should be compensated by that in astronomy, due to its complexity, historical inconsistency and limited applicability.
\end{abstract}

Keywords: tide, principia, education, astronomy, oceanography

\section{Introduction}

The equilibrium theory of tides by Newton ${ }^{\dagger}$ (1686) states that, on the Earth, two opposite-sided oceanic bulges are formed along the line connecting centers of the Earth and the source of gravity (e.g. the Moon or the Sun). According to this theory, tides on the Earth is a phenomenon driven mainly by differential gravity contributed by the Sun and the Moon. The proximity of the Moon relative to the Sun makes the contribution by the former larger than the latter roughly by a factor of two. Although this theory successfully explains the period of semidiurnal tides (two high tides and two low tides per day) as well as the spring tides and neap tides, the dynamic theory of tides which incorporates the hydrodynamic effect of the fluid as well as the Earth's spin was first introduced by Laplace (1775) who attempted to explain diurnal tides, mixed tides, and the amplitude

*Corresponding author: kjahn@chosun.ac.kr

Tel: 82-62-230-7340

Fax: 82-62-230-7935 of tides observed in reality. At any rate, because tide can change the height and dynamics of the ocean both locally and globally, it is an important phenomenon in oceanography; therefore, a well-established teaching method of this phenomenon is crucial. In astronomy, this is even more crucial because tides occur on any astronomical systems with finite size, which naturally span a huge dynamic range: satellites, planets, stars, black hole accretion disks, star clusters, and galaxies.

Tides in the earth science (education) department is usually taught in an inter-disciplinary approach: it is taught both in oceanography and astronomy courses at college level. Usually, the concept of tides is extensively explained in college-level oceanography courses up to the dynamic theory, while in college-level astronomy courses only up to the equilibrium theory. Nevertheless, there is an overlap in both disciplines in that the equilibrium theory of tides is taught at least. The

\footnotetext{
"We also refer to Chandrasekar (1995), a version translated and interpreted into English and the modern scientific language, and Cho (1999), a version translated into Korean, when we need to refer to Principia, because the original transcript by Newton, written in Latin, is illegible by us other than figures.
} 
usual textbooks used in this context are by Garrison (2007, 2008) and Knauss (1998) in oceanography, and by Zeilik et al. (1992) and Karttunen et al. (2008) in astronomy, to name only a few. Throughout this paper, we restrict our attention to these widely used textbooks and assume that the descriptions used in these textbooks represent general textbook descriptions of tides in oceanography and astronomy, respectively, although different textbooks and methods may be used in various individual institutions.

According to these oceanography textbooks (Garrison, 2007, 2008; Knauss, 1998), how the equilibrium theory of tides is explained in oceanography differs from the usual treatment in astronomy. In general, the description used in oceanography seems more complicated than that in astronomy. The former is based upon the reference frame fixed to the Earth, which is a non-inertial frame, while the latter uses an approximately inertial lab frame fixed to the Sun in which both the Moon and the Earth are observed to have motions. The former introduces the center of mass of the Earth and the Moon system (if one considers the tides by the Moon), while the latter does not. Furthermore, the former introduces the centrifugal force due to the non-inertial nature of the reference frame, while the latter does not. Overall, the usual treatment of tides in oceanography has a potential to generate more confusion among students than the treatment in astronomy.

This paper is intended to inform some educators of earth sciences, who may be unaware of the discrepancy in the textbook description of tides between astronomy and oceanography. This attempt will inform some astronomers of the current textbook description of tides in oceanography, and promote their communications with oceanographers on this issue. To support this, we provide various astrophysical examples as well as an important historical background, which will guide some readers to reconsider their teaching methods of tides, if they are only familiar with the conventional textbook description of tides in oceanography. From this point on, the oceanography textbook description of the equilibrium theory of tides is denoted by description $\mathrm{O}$, while that in astronomy by description A. Throughout this paper, the Earth is assumed to be composed of a rigid body in perfect spherical symmetry and an oceanic fluid which can respond to gravity instantaneously without any real hydrodynamic effects. The discussion is also restricted to the tide by the Moon when reviewing the textbook descriptions in section "Textbook descriptions of the Equilibrium Theory of Tides," as is the case of Garrison (2007, 2008). We may loosely mix the notion of force with that of acceleration: force in our paper means force per unit mass, unless specified otherwise.

While preparing this paper, we have realized that some of our view is already shared by an internet article by Simanek (2009). Our paper, however, provides a full quantitative argument along with a historical background and various astrophysical examples. Simanek (2009) asserts that the centrifugal force is a misconception, but we discuss in section "Summary and Discussion" that sometimes noninertial frames are favored over inertial reference frames and that the concept of fictitious force becomes necessary. We also limit our focus only to the higher-level education in US and Korea (see for example Lee 2006 for some misconceptions on tides in the middle-level education in Korea), but when our future investigations mature, we may identify whether the same problem exists more generally in other foreign educational systems as well.

\footnotetext{
This is only approximately true, because the solar system revolves around the center of the Milky Way. The corresponding centrifugal acceleration, $a_{c}=\frac{v^{2}}{R}$, is only about $2 \times 10^{-10} \mathrm{~m} / \mathrm{s}_{2} \approx 2 \times 10^{-11} \mathrm{~g}$, where $\mathrm{v} \approx 220 \mathrm{~km} / \mathrm{s}$ is the rotational velocity of the Sun relative to the center of the Milky Way, $R \approx 8.5 \mathrm{kpc}$ is the distance from the center of the Milky Way to the Sun, and $\mathrm{g}=9.8 \mathrm{~m} / \mathrm{s}^{2}$ is the gravitational acceleration due to the mass of the Earth. This is even much smaller than the tidal acceleration on the Earth by the Moon, which is about $10^{-7} \mathrm{~g}$, and therefore the Sun-fixed frame may be regarded as an inertial frame for the sake of our discussion.
} 
Table 1. Introductory oceanography courses in some prestigious US institutions which adopt Gross $(1997,1998)$ as the primary textbook. This survey is not complete, because it covers only those courses whose syllabi are publicly available. Nevertheless, it shows that Gross $(1997,1998)$ is among the most used textbooks in introductory oceanography lectures. The list is sorted in the alphabetical order of the name of institutions

\begin{tabular}{lll}
\hline \hline \multicolumn{1}{c}{ Institution } & \multicolumn{1}{c}{ Lecturer } & \multicolumn{1}{c}{ Course/term } \\
\hline City University of New York & Buonaiuto, F. & Introduction to Oceanography/Fall 2009 \\
Johns Hopkins University & Haine, T.; Del Castillo, C. & Introductory Oceanography/Fall 2009 \\
Mississippi State University & McNeal, K. & Ocean Science/Summer 2008 \\
Northwestern University & Blair, N. & The Ocean, the Atmosphere and Our Climate/Fall 2009 \\
Ohio State University & Grottoli, A. G. & Introductory Oceanography/Fall 2009 \\
Rutgers University & Rona, P. A. & Introduction to Oceanography/Fall 2009 \\
University of California at Berkeley & Powell, Z.; Bishop, J. & Introduction to Oceans/Fall 2007 \\
University of California at Santa Cruz & Griggs, G. & Oceanography Earth Sciences 1/Summer 2009 \\
University of Colorado & Marchitto, T. & Introduction to Oceanography/Spring 2009 \\
University of Florida & Zimmerman, A. & Introduction to Oceanography/Fall 2007 \\
University of Georgia & Ketter, C. T. & Marine Science for Teachers/Summer 2006 \\
Washington State University & Rollwagen-Bollens, G. & Introduction to Ocean Science/Spring 2008 \\
\hline
\end{tabular}

\section{Textbook Descriptions of the Equilibrium Theory of Tides}

\section{Description O: Equilibrium theory of tides in non-inertial frame, taught in oceanography}

We refer to the description used in Garrison (2007, 2008) and Knauss (1998), which are widely used college-level textbooks in Korea, especially in the earth science education department. They may be treated as representative textbooks of oceanography in US as well, as Garrison (2007, 2008), for example, is currently adopted as the primary textbook in many US institutions (Table 1). Among the many introductory oceanography textbooks, other widely used ones as alternatives are Gross (1995), Trujillo and Thurman (2007), Stowe (1996), Sverdrup, Duxbury and Duxbury (2005), etc. A more thorough investigation of the oceanography curricula of the earth science (education) departments in individual institutions seems necessary, but the fact that Garrison (2007, 2008 ) is one of the most used college-level textbooks is sufficient for us to raise this issue, and therefore we delay such an investigation to the future.

Garrison $(2007,2008)$ uses the reference frame fixed to the Earth, so it is a rotating reference frame, which in turn is a non-inertial frame. The rotation is somewhat peculiar because different points on the Earth have different centers of rotation (Fig. 1.; see also Garrison, 2007, 2008). The center of the Earth rotates around the center of mass of the Earth-Moon system, located at distance

$$
R_{C O M} \equiv \frac{m_{M}}{m_{E}+m_{M}} d
$$

from the center of the Earth, where $m_{M}$ is the mass of the Moon, $m_{E}$ the mass of the Earth, and $d$ the distance from the center of the Earth to the center of the Moon. If the daily rotation of the Earth is neglected, each point on the earth's rigid body rotates around its own center with radius $R_{\text {Сом. }}$. In the Earthfixed frame, this induces a fictitious acceleration, the centrifugal acceleration directed parallel to line EM and away from the Moon, whose amplitude is given by

$$
a_{c} \equiv\left|\vec{a}_{c}\right|=R_{\text {COM }} \omega^{2},
$$

where $\omega$ is the angular velocity of the Earth-Moon system. Because daily and seasonal variation of $d$ is negligible (both the Earth and the Moon revolve around their center of mass in circular orbit), $d, R_{\text {COM }}$, and $\omega$ are all constants. $\omega$ is given by the Kepler's third law on the binary system:

$$
\omega^{2}=\frac{G\left(m_{M}+m_{E}\right)}{d^{3}} .
$$




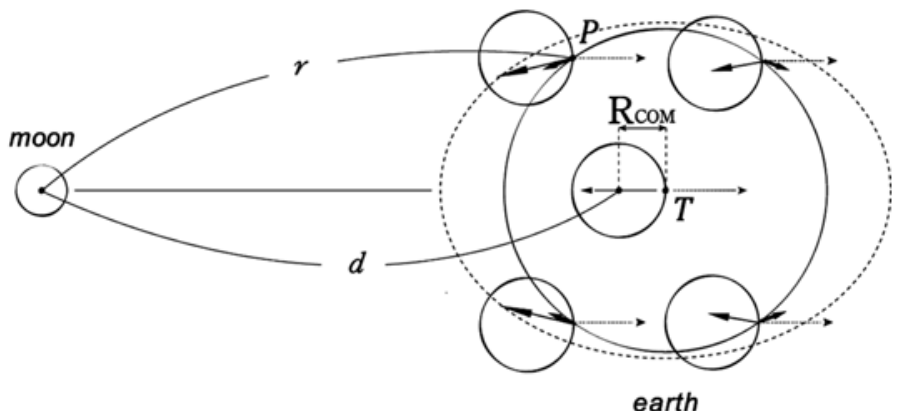

Fig. 1. How the equilibrium theory of tides is described in college-level oceanography textbooks (e.g. Garrison 2007, 2008), adopting a reference frame fixed to the Earth. The whole rigid body as well as the center of mass (point $\mathrm{T}$ ) of the Earth undergoes a circular motion shown as five identical circles on the right, with radius $R_{C O M}=\frac{m_{M}}{m_{E}+m_{M}} d$. The centrifugal force on every point of the Earth, therefore, is identical in both amplitude and direction (thin dotted arrows). Meanwhile, the Moon attracts each point on the Earth with force vectors depicted by solid thin arrows. One can obtain the tidal force on point $\mathrm{P}$ or other places, for example, by the summation of these two vectors, resulting in the net force vectors (thick solid arrows).

Equations (2) and (3) give

$$
a_{c}=\frac{G m_{M}}{d^{2}}
$$

whose amplitude is equivalent to the gravitational pull felt by the center of the Earth due to the lunar mass.

The next step is straightforward. At any point of interest on the surface of the Earth, one can calculate the gravitational pull (acceleration) by the Moon (let us denote this by $\vec{a}_{P M}$ ):

$$
a_{P M} \equiv\left|\vec{a}_{P M}\right|=\frac{G m_{M}}{r^{2}},
$$

which is directed toward the Moon, and where $r$ is the distance from the point of interest to the Moon. Finally, one can obtain the tidal acceleration (denoted by $\vec{a}_{t, O}$ where the subscript $\mathrm{t}$ represents tidal force, and $\mathrm{O}$ description $\mathrm{O}$ ) as follows:

$$
\vec{a}_{t, O}=\vec{a}_{P M}+\vec{a}_{c} .
$$

Knauss (1998) adopts a similar approach, also by specifically mentioning the centrifugal force. Both Garrison (2007, 2008) and Knauss (1998) lack some details described in equations (1) through (6). For example, Garrison $(2007,2008)$ specifies none of the amplitudes of $\vec{a}_{t, O}, \vec{a}_{P M}$ and $\vec{a}_{c}$ but only describe them in qualitative way, while Knauss (1998) specifies amplitudes of these forces but skip equations (1) and (2). We believe that students should be well informed about all these steps for a thorough understanding when one intends to teach tides using description $\mathrm{O}$.

First note that equation (6) is a vector summation, not a subtraction (see Fig. 1). Because the fictitious force (per unit mass) $\vec{a}_{c}$ is "felt" by the ocean in this reference frame, one should treat $\vec{a}_{c}$ as if it is a real force and add to any other existing force, such as $\vec{a}_{P M}$. Note also that $\vec{a}_{c}$ in equation (4) does not show an explicit dependence on $R_{C O M}$ (or a term like $m_{M}+m_{E}$ ). A wise student might wonder why the information on the center of mass of the Earth-Moon system, represented by $R_{C O M}$, is lost in the final result in this approach. Indeed, description A gives a clear answer to this question, because the concept of the center of mass is unnecessary in the inertial frame, as will be described in the following subsection.

Description A: Equilibrium theory of tides in inertial frame, taught in astronomy

As mentioned earlier, the equilibrium theory of tides in astronomy adopts an inertial reference frame. In an inertial frame, by definition, there exist no fictitious forces such as the centrifugal force or Coriolis force. All that is required is to account for the gravity given 


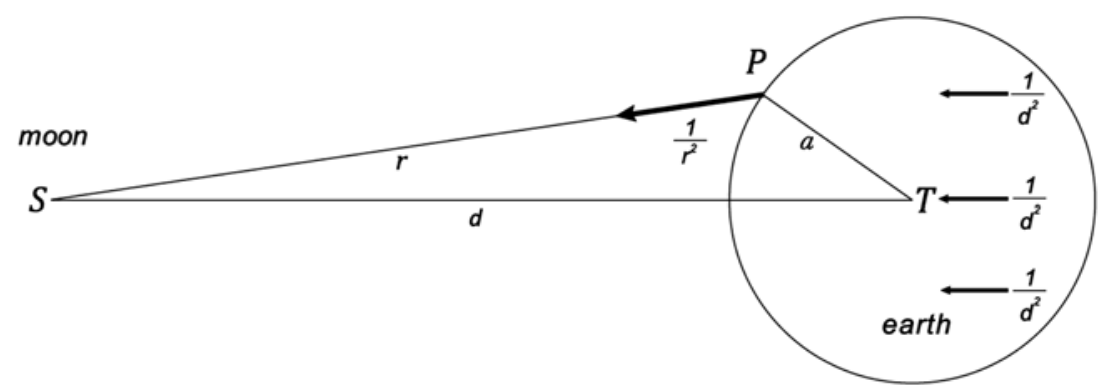

Fig. 2. How the equilibrium theory of tides is described in college-level astronomy textbooks (e.g. Zeilik et al. 1992). adopting an (almost) inertial frame fixed to the Sun. The Moon at point $\mathrm{S}$ attracts the ocean at point $\mathrm{P}$ and the rigid body of the Earth with force (in units of $G m_{M}$ ) and $1 / r^{2}, 1 / d^{2}$ respectively. Their directions are also clarified in the figure. The tidal force on point $\mathrm{P}$ is then given by vector subtraction of the latter from the former, given by equation (9).

by the source mass and its distance.

In Zeilik et al. (1992), one of the representative college-level astronomy textbooks, the theory is described in terms of the differential gravity in the following way (see also Figure 2). At any point of interest on the surface of the Earth, one can calculate the gravitational acceleration $\vec{a}_{P M}$ by the Moon, in the same way as in description $O$. Its amplitude is given by

$$
a_{P M} \equiv\left|\vec{a}_{P M}\right|=\frac{G m_{M}}{r^{2}},
$$

which is directed toward the Moon. In the meantime, the gravitational acceleration of the center of the Earth, $\vec{a}_{E M}$, has an amplitude

$$
a_{E M} \equiv\left|\vec{a}_{E M}\right|=\frac{G m_{M}}{d^{2}}
$$

and is directed parallel to line EM and toward the Moon. Because the Earth is a rigid body (except the ocean), any part of the rigid body is accelerated universally at $\vec{a}_{E M}$ (when neglecting the daily rotation of the Earth). Finally, the differential acceleration of the ocean and the surface of the Earth, $\vec{a}_{t, A}$ (where the subscript $t$ represents tidal force, and A description A), is given as

$$
\vec{a}_{t, A}=\vec{a}_{P M}-\vec{a}_{E M} .
$$

This is the tidal acceleration bearing the notion of differential gravity, as is clearly seen in equation (9). $\vec{a}_{t, A}$ is of course numerically identical to $\vec{a}_{t, O}$ obtained by description $\mathrm{O}$ (equation 6 ), while their interpretations differ significantly. This difference is shown clearly from how two accelerations are added in equations (6) and (9): the former is in summation while the latter is in subtraction. Karttunen et al. (2008) adopts a similar approach.

There are other preferable features in description A. Note how succinct it is to derive the final result, both numerically and conceptually. All that is required is to calculate different forces (per unit mass) at different places, by a single mass source of interest (or by more sources, if any, by simple linear superposition of forces). It is also easy to understand how bulges form at two opposite sides. The fluid close to the Moon will move faster than the rigid body of earth, while the fluid farthest from the Moon will move slower than the rigid body of earth and lag behind, and thus forming bulges in both places. The symmetry of bulges is a result of largeness of $d$ relative to $a$, such that all quantities can be expanded quite accurately in the linear order of $a / d$ (e.g. Zeilik et al., 1992).

\section{Newton's equilibrium theory of tides in Principia}

We now examine how Newton developed the equilibrium theory of tides in Philosophiae Naturalis Principia Mathematica (Principia in short; inarguably 


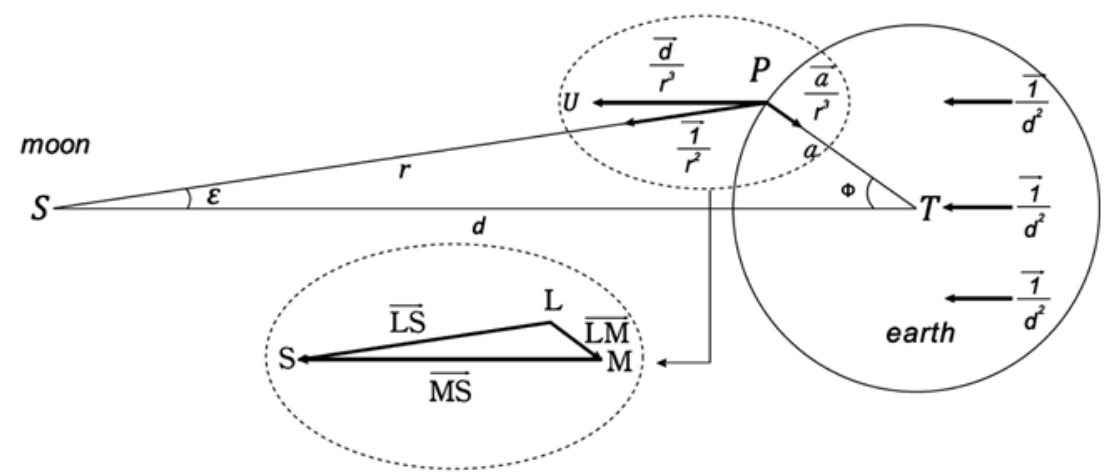

Fig. 3. Force triangle used to describe tides by Newton. The original figure in Principia (1686) overlays the force triangle (LSM) on top of the distance triangle (PST). Newton enforced the force triangle to be in a similar form with the distance triangle. Therefore, force $\overrightarrow{L S}=\frac{\overrightarrow{1}}{r^{2}}$ has the real physical origin, while $\overrightarrow{M S}=\frac{\vec{d}}{r^{3}}$ and $\overrightarrow{L M}=\frac{\vec{a}}{r^{3}}$ are just derived quantities. $\frac{\overrightarrow{1}}{d^{2}}$ is the acceleration of the whole rigid body of the Earth, whose origin is again by the Moon. Vector notations here follow those of Chandrasekar (1995).

one of the greatest masterpieces in the history of physics), and compared this with description $\mathrm{O}$ and description A to see which one is more consistent with Newton's original description. As noted earlier, we refer to legible, translated versions by Chandrasekar (1995) and Cho (1999), while some part of Fig. 3 is extracted from the original Principia (1686). Somewhat unfamiliar vector notations in this section follow those of Chandrasekar (1995).

Before treating tides, Newton developed a perturbation theory of a three-body system, where one heavy object attracts light objects by gravity, under Proposition LXVI (see pages 236-264, especially figures on pages 238-239 of Chandrasekar, 1995, and pages 316-334 of Cho, 1999) in the Book I of Principia. When object $\mathrm{P}$ (light) revolves around object $\mathrm{T}$ (heavy) without any disturbance from other objects, object $\mathrm{P}$ should follow an elliptical trajectory. However, when another light object, object S, exists somewhere far from the P-T system, the motion of object $\mathrm{P}$ will be disturbed and will no longer follow the elliptical trajectory (Fig. 3). In developing this theory, Newton decomposed the acceleration vector (in units of $G m_{M}$, where $m_{M}$ is the mass of object $\mathrm{S}$ ) by object $\mathrm{S}$ on object $\mathrm{P}, \overrightarrow{L S}$, into two components which are not orthogonal to each other, namely $\overrightarrow{L M}$ and $\overrightarrow{M S}$ (Fig. 3). As interpreted by Chandrasekar (1995) on page 238, because Newton designed $\overrightarrow{L M}$ to be parallel to the actual spatial line PT, $\overrightarrow{L M}$ does not induce a torque to object P. Thus, $\overrightarrow{L M}$ does not change the law of areas (or Kepler's 2nd law), while both $\overrightarrow{L M}$ and $\overrightarrow{M S}$ contribute to the departure of object $\mathrm{P}$ from the elliptical trajectory (Chandrasekar, 1995). Note that both $\overrightarrow{L M}$ and $\overrightarrow{M S}$ are contributed by the source mass $\mathrm{S}$, which are simply derived quantities from the geometry only, once we identify the vector $\overrightarrow{L S}$ as the true force (per unit mass) acted on object $\mathrm{P}$ by object $\mathrm{S}$.

Newton then applied the same geometry and force calculation to the problem of tides. Now, if one is interested in the tides by the Moon, object S, T, and $\mathrm{P}$ can be identified as the Moon, the Earth, and a point on the surface of the Earth, respectively. Newton then obtained the differential gravity in direction PU (from $\mathrm{P}$ to $\mathrm{U}$ ) by subtracting the acceleration of the Earth as follows,

$$
G m_{M}\left(|\overrightarrow{M S}|-\left|\frac{\overrightarrow{1}}{d^{2}}\right|\right)=G m_{M}\left(\frac{d}{r^{3}}-\frac{1}{d^{2}}\right),
$$

while allowing the radial force per unit mass (in units of $G m_{M}$ ) by the moon, $\overrightarrow{L M}=\frac{\vec{a}}{r^{3}}$, to contribute later in the radial direction PT (Fig. 3). Newton then calculated the radial (direction PT) component of the net tidal force by including the contribution by $\overrightarrow{L M}$, 
and the transverse (tangential to the Earth's surface) component as well. It is evident that in the end, Newton's approach, if written in vector summation, becomes identical to equation (9), because when $\overrightarrow{L M}$ is accounted for in obtaining the net differential gravity, it yields

$G m_{M}\left(\overrightarrow{L M}+\overrightarrow{M S}-\frac{\overrightarrow{1}}{d^{2}}\right)=G m_{M}\left(\overrightarrow{L S}-\frac{\overrightarrow{1}}{d^{2}}\right)=G m_{M}\left(\frac{\overrightarrow{1}}{r^{2}}-\frac{1}{d^{2}}\right)$

We refer readers to equations (1) through (4) under section 112 (page 402) of Chandrasekar (1995) for the original approach by Newton. Equation (11) is our own interpretation, but this proves that Newton's result and approach are implicitly identical to those of equation (9).

Aside from some difference in steps leading to equations (9) and (11), which merely comes from the choice of vector directions, reasonings behind these equations are consistent with each other. Newton adopted an inertial frame as is done in today's astronomy textbooks: in deriving equation (10), and implicitly equation (11), Newton used neither the concept of center of mass nor the centrifugal force. Therefore, even in the historical viewpoint, the textbook description in astronomy is favored against that in oceanography if one seeks consistency with the original work by Newton.

\section{Tides in Astrophysical phenomena: Description A vs. Description O}

Tides occur on any physical system with finite size when external mass sources exist. It has the effect of stretching a given system, and this is usually referred to as "tidal force" in astronomy. Following the reasoning behind description $\mathrm{A}$, it is easy to perceive the fact that tidal force originates from differential gravity.

It is no wonder, therefore, that tidal effects are a common phenomenon in astrophysics. Tidal tail formation, which refers to the formation of thin, curved structure of mass stretched out from a disrupted galaxy, as a result of a galaxy merger event, is one among the notable examples. Tidal stretching of a finite-size system near a black hole is another. Star clusters can sometimes be disrupted by nearby masses. These are only a few examples among various astrophysical phenomena related to tides. Let us treat only tidal tail formation and tidal force near a black hole, and demonstrate how inappropriate description $\mathrm{O}$ becomes in these cases.

\section{Tidal tail formation}

When two or more galaxies have a close encounter with some impact parameter, the inner structure of each galaxy is altered due to the gravity contributed by other galaxies. Depending on physical parameters such as the impact parameter, relative velocities, directions of spins, size, and mass of galaxies, the fate of this encounter can have different degrees and forms: mild deformation, some mass loss, tidal tail formation, vigorous heating and deformation, or even a total disruption.

The tidal tail is one result arising from such galaxy interaction events. When two galaxies interact in proximity, differential gravity from each causes tidal force inside each galaxy. Sometimes, disrupted material due to this tidal force forms ring shaped structure and is gradually detached from the galaxy. When tidal force is large, part of the ring which has been pushed away from the disturbing galaxy can form a thin, distinctly curved structure, called the tidal tail (e.g. Binney and Tremaine, 1988).

Theorists have reproduced tidal tail mostly through numerical simulations (e.g. Toomre and Toomre, 1972). Simulating galaxy dynamics requires calculating both collisionless N-body dynamics and hydrodynamics (collisional between particles) in general. When hydrodynamic effect becomes negligible, however, simple N-body simulation becomes sufficient. Even in doing so, one still needs to numerically integrate the equation of motion of each particle,

$$
\frac{d^{2} \vec{x}_{i}}{d t^{2}}=-\sum_{j \neq i} \frac{G m_{j}}{\mid \overrightarrow{x_{i}-\left.x_{j}\right|^{2}}} \hat{r}_{i j}
$$


where a particle $i$ located at $\vec{x}_{i}$ is attracted through gravity by each and every other particle $j$ located at $\vec{x}_{j}$, whose mass is $m_{j}$, and $\hat{r}_{i j}$ is a unit vector directed from particle $j$ to $i$. The numerical domain where particle motions are calculated, usually called the simulation box, is an inertial reference frame, and equation (12) is the single differential equation to integrate. Also, from equation (12), one can easily calculate the tidal force between particle 1 and particle 2:

$$
\vec{a}_{1}-\vec{a}_{2}=-\left(\sum_{j \neq 1} \frac{G m_{j}}{\mid \overrightarrow{x_{1}-\left.\vec{x}_{j}\right|^{2}}} \hat{r}_{1 j}-\sum_{j \neq 2} \frac{G m_{j}}{\mid \overrightarrow{x_{2}-\left.\vec{x}_{j}\right|^{2}}} \hat{r}_{2 j}\right),
$$

as in equation (9).

Note that numerical simulations of interacting galaxies do not consider a reference frame fixed to one single galaxy among two or more interacting galaxies, but just an inertial frame (simulation box) as description A does. In contrast, description $\mathrm{O}$ adopts a frame fixed to the Earth, where gravity is contributed by two objects, the Moon and the Earth. One could, in principle, adopt such a non-inertial frame in simulating interacting galaxies, but this would induce a fictitious force term which has to be added to equation (12) and make the simulation consume more CPU (central processing unit) time. Moreover, it becomes even impossible to define such a reference frame when matter from one galaxy flows into the other; one then cannot have a clear separation of objects from one another, and the center of mass of one galaxy can hardly be defined.

This example also indicates that description $\mathrm{A}$ is favored even when calculating the tidal force in between oceanic fluid elements alone (or that inside the Earth alone). In description A, one can simply calculate differential gravity in a similar form of equation (13) (where the dominating mass source causing the tidal force is the Moon), while in description $\mathrm{O}$, one has to calculate the centrifugal forces of each fluid element, which may or may not be equal to each other. description $\mathrm{O}$ becomes more complicated as one considers any deviations from the specific case of the oceanic tide against the Earth.

\section{Tidal force near a black hole}

The black hole is an object usually formed by a collapsing massive star. When formed, a black hole distorts the spacetime around it nothing like other astrophysical objects. An exact solution to its spacetime metric was discovered by Schwarzschild (e.g. Misner et al., 1973). For simplicity, we only consider a black hole with neither spin nor charge.

Consider a free-falling astronaut into a black hole. The astronaut may move into the black hole with null impact parameter, namely straight toward the black hole. Let us further assume that the astronaut is undergoing a head-on infall, namely his head directed toward the black hole, while his feet away from the black hole. In an inertial reference frame, one can easily calculate the tidal force between his head and feet as,

$$
a_{h}-a_{f}=\frac{G m_{B H}}{R_{B h}^{2}}-\frac{G m_{B H}}{R_{B f}^{2}},
$$

where $m_{B H}$ is the mass of the black hole, and $a_{h}, a_{f}$, $R_{B h}, R_{B f}$ are acceleration of head toward the black hole, acceleration of feet toward the black hole, distance between the black hole and the head, and distance between the black hole and the feet, respectively. When the astronaut is too close to the black hole, tension inside body cannot balance the tidal force and therefore his body will tear apart (note that we just work under the regime where Newtonian approximation is valid, far beyond the Schwarzschild radius, for simplicity).

Equation (14) is easily derived in an inertial reference frame. What if we consider a reference frame fixed to the center of mass of the astronaut? In this specific example, because the system is not rotating, there is no centrifugal force. description $\mathrm{O}$ fails miserably in this specific example in explaining the tidal force felt by the astronaut. To salvage description $\mathrm{O}$, one should instead realize that there exists another fictitious force, the inertial force, felt by the feet which comes from inertia of the feet that tends to stay still against the head. And the strength of this inertial force must be equal to $\frac{G m_{B H}}{R_{B f}^{2}}$, which 


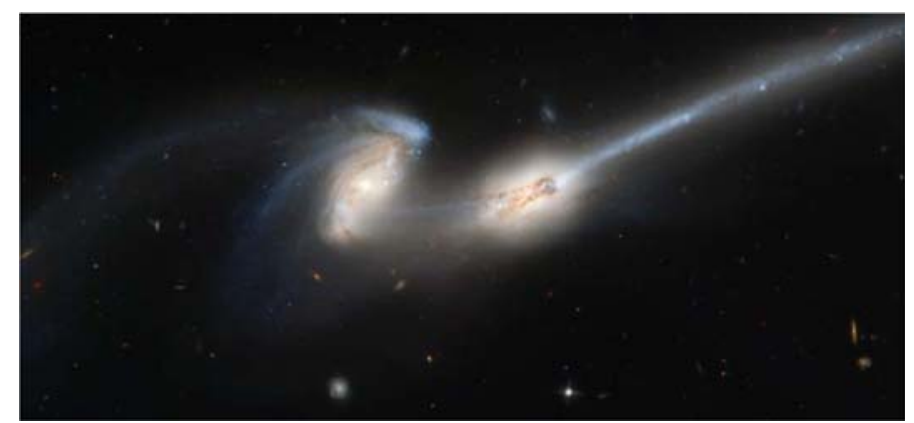

Fig. 4. Tidal tail formed in a pair of interacting galaxies NGC 4676, nicknamed "The Mice." The tail, stretched away from the galaxy on the right (NGC 4676A) toward the top right corner of the picture, is composed of material stripped away from NGC 4676A due to the tidal force caused by the galaxy on the left (NGC 4676B). In the tail, some of the material is being converted into stars. This picture is an excerpt from the hubblesite.org (2009), under the permission specified in its copyright notice, and credited to NASA, H. Ford (JHU), G. Illingworth (UCSC/LO), M. Clampin (STScI), G. Hartig (STScI), the ACS Science Team, and ESA.

should be justified by Newton's law of action and reaction. Description O brings in some complexity in the analysis.

Therefore, description $\mathrm{A}$ is again favored against description $\mathrm{O}$ in the case where rotation is not involved. Examples similar to this configuration are a body standing on the surface of a neutron star, galaxies colliding head-on, etc.

\section{Summary and Discussion}

We have argued that the conventional textbook description of the equilibrium theory of tides in oceanography (description $\mathrm{O}$ ) is far too complicated and limited, and that in astronomy (description A) is more favored in general. We have also shown that the original derivation of the theory by Newton in Principia is consistent with description A. Difference between these descriptions comes from the choice of reference frames: description A adopts an inertial frame which does not have any fictitious forces, while description $\mathrm{O}$ adopts a non-inertial frame where fictitious forces have to be considered. Various astrophysical tides are most simply explained by description A, while not as such by description $O$. In many astrophysical phenomena, description $\mathrm{O}$ becomes even inapplicable, as demonstrated by the case of tidal tail formation when galaxies interact strongly. In terms of ease of understanding, ease of interpretation, historical consistence and extendibility to other tidal phenomena, method A is preferable to method O.

Is non-inertial frame always disfavored? Of course not. When the spin (daily rotation) of the Earth is considered, the non-inertial frame is favored against the inertial frame, in order to treat Coriolis force, a fictitious force caused by the Earth's spin. One could in principle solve everything in an inertial frame, in which the earth is observed to rotate daily. However, to describe the physics of typhoon, for example, it is more convenient to adopt the notion of Coriolis force in the Earth-fixed reference frame than to describe everything in the inertial reference frame. The dynamic theory of tides by Laplace is affected by both gravity by exterior sources (the Moon and the Sun) and the spin of the Earth. In the dynamics theory of tides, therefore, the non-inertial reference frame is more convenient. There may be cases where different reference frames have both advantages and disadvantages over each other and one has to choose the most convenient reference frame. Numerical simulation of meteorological or oceanic phenomena (e.g. Park and An, 1990; Park and Cho, 1999), when hydrodynamic interaction with the rigid body of Earth becomes important, might benefit from the Earth-fixed frame even in the presence of gravitational effects from exterior sources. One could then easily calculate 
fluid dynamics on a given computational mesh, for example, fixed to the Earth, with no need to resolve the motion of the Earth itself.

Nevertheless, the equilibrium theory of tides is best described in an inertial reference frame, as is the case of description A. It is somewhat striking that description $\mathrm{O}$ is adopted in oceanography textbooks and lectures even when the equilibrium theory, not the dynamical theory, is explained. Even though our work is based upon a limited range of textbook descriptions, description $\mathrm{O}$ seems to be adopted on a more general basis even now. Most of the representative collegelevel oceanography textbooks adopt description $\mathrm{O}$, as are the cases with Garrison (2007, 2008), Knauss (1998), Gross (1995), etc.

The basic concept of description $\mathrm{O}$ is seemingly a required knowledge for college students. For example, a question has appeared in a national test recently which asks the notion of description $\mathrm{O}$ (problem 25 of the Earth Science Major, Korean Middle and High School Faculty Employment Test, year 2005). We worry that this description can easily generate confusion among students and even cause misconceptions that tides occur only on rotating system and Newton's original development of the theory is consistent with description $\mathrm{O}$. Therefore, we argue that description $\mathrm{O}$ should be scrutinized by the oceanography community at this point. Even though it is still premature to suggest a concrete curriculum or teaching methods to reconcile these two different approaches, we suggest that at least description $\mathrm{A}$ is taught in oceanography lectures at the same depth as description $\mathrm{O}$, and that the historical background described in this paper is treated both in oceanography and astronomy lectures. It would also be beneficial if astronomy lectures treat description $\mathrm{O}$ as well, and let students make a fair comparison between the two approaches. This would then help to strengthen the understanding of the equilibrium theory of tides by college students.

\section{Acknowledgment}

We thank Junghoon Kim for helping us identify oceanography textbooks referred here, and Jongwon Um for the help with figures. This study was supported by research funds from Chosun University, 2008.

\section{References}

Binney, J. and Tremaine, S., 1988, Galactic Dynamics. Princeton University Press, Princeton, USA, 747 p.

Chandrasekar, S., 1995, Newton's Principia for the Common Reader. Oxford University Press, NY, USA, 616 p.

Cho, K., 1999, Principia 1 (translated version of Newton's Principia Book I into Korean). SeoHae Miscellany, Seoul, Korea, 425 p.

Simanek, D.E., Misconceptions on Tides: http://www.lhup. edu/ dsimanek/scenario/tides.htm (retrieved on Jul 25, 2009)

Garrison, T., 2007, Essentials of Oceanography, 3rd Edition. Korean Translation Edition by Lee, S., Kang, H., Kim, D., Lee, D., Lee, J., Chung, I., and Heo, S., Sigma Press Inc., Seoul, Korea, 389 p.

Garrison, T., 2008, Oceanography: An Invitation to Marine Science, 3rd Edition. Korean Translation Edition by Kang, H., Kim, D., Lee, D., .Lee, S., Lee, J., Chung, I., and Heo, S., Sigma Press Inc., Seoul, Korea, 537 p.

Gross, M.G., 1995, Principles of Oceanography, 7th Edition. Prentice Hall, NJ, USA, 240 p.

Hubblesite.org: http://hubblesite.org/gallery/album/galaxy/ pr2002011d (retrieved on July 25, 2009)

Knauss, J.A., 1998, Introduction to Physical Oceanography. Korean Translation Edition by Ahn, H. and Oh, I. Sigma Press Inc., Seoul, Korea, 324 p.

Laplace, P.S., 1775, Recherches sur Plusieurs Points du Systeme du Monde. Memoires de l'Academie Royale des Sciences de Paris, 88, 75-182.

Lee, K.-Y., 2006, Investigation of Mental Models about Tide for Scientifically Talented Middle School Students by Analyzing Facet of Conceptual Types by Context. Journal of Korean Earth Science Society, 27, 6-14. (in Korean)

Misner, C.W., Thorne, K.S., and Wheeler, J.A., 1973, Gravitation. Freeman, San Francisco, USA, 1279 p.

Newton, I., 1686, Philosophiae Naturalis Principia Mathematica, Book I. the Royal Society, London, England, $511 \mathrm{p}$.

Park, K.-A. and An, H.-S., 1990, A Study of Numerical Experiments on the Tide and Surge in the Korea Straits. Journal of Korean Earth Science Society, 11, 13-20. (in Korean)

Park, Y.-W. and Cho, Y.-K., 1999, Numerical Experiment on the Tide in Hampyung Bay, West Cost of Korea. 
Journal of Korean Earth Science Society, 20, 380-387. (in Korean)

Stowe, K., 1996, Exploring Ocean Science, 2nd Edition. John Wiley and Sons, NJ, USA, $190 \mathrm{p}$.

Sverdrup, K., Duxbury, A. and Duxbury, A., 2005, Fundamentals of Oceanography, 5th Edition. McGraw-Hill, NY, USA, $352 \mathrm{p}$.

Trujillo, A. and Thurman, H.V., 2007, Essentials of Oceanography, 9th Edition. Prentice Hall, NJ, USA, 576 p.

Toomre, A. and Toomre, J., 1972, Galactic Bridges and
Tails. Astrophysical Journal, 178, 623-666.

Zeilik, M., Gregory S.A., and Smith E.V., 1992, Introductory Astronomy and Astrophysics, 3rd Edition. Sunders College Publishing, Fort Worth, USA, $520 \mathrm{p}$.

Karttunen, H., Kroger, P., Oja, H., Poutanen, M., and Donner, K.J., Fundamental Astronomy, 5th Edition. Korean Translation Edition by Kang, H., Kim, S., Min, Y., Yun, H., Lee, S., Chang, H., and Hong, S., Sigma Press, Seoul, Korea, 588 p.

Manuscript received: August 3, 2009

Revised manuscript received: August 31, 2009

Manuscript accepted: September 15, 2009 